Research Paper

\title{
YSY01A, a Novel Proteasome Inhibitor, Induces Cell Cycle Arrest on G2 Phase in MCF-7 Cells via ERa and $\mathrm{PI} 3 \mathrm{~K} / \mathrm{Akt}$ Pathways
}

\author{
Bingjie Xue ${ }^{1}$, Wei Huang1, Xia Yuan¹, Bo Xu², Yaxin Lou³, Quan Zhou'1, Fuxiang Ran¹, Zemei Ge4, Runtao \\ $\mathrm{Li}^{4 凶}$, and Jingrong Cui ${ }^{\bowtie}$ \\ 1. State Key Laboratory of Natural and Biomimetic Drugs, School of Pharmaceutical Sciences, Peking University, 100083, Beijing, China; \\ 2. Instrumental Analysis Center of State Key Laboratory of Natural and Biomimetic Drugs, School of Pharmaceutical Sciences, Peking \\ University, 100083, Beijing, China; \\ 3. Lab of Proteomics Medical and Healthy Analytical Center, Peking University, Beijing, China; \\ 4. Peking University School of Pharmaceutical Sciences Department of Medicinal Chemistry, Beijing, China
}

$\bowtie$ Corresponding authors: Jingrong Cui, Email: jrcui@bjmu.edu; Runtao Li, Email: lirt@bjmu.edu.cn.

(C) 2015 Ivyspring International Publisher. Reproduction is permitted for personal, noncommercial use, provided that the article is in whole, unmodified, and properly cited. See http://ivyspring.com/terms for terms and conditions.

Received: 2014.10.05; Accepted: 2014.11.15; Published: 2015.02.06

\begin{abstract}
Given that the proteasome is essential for multiple cellular processes by degrading diverse regulatory proteins, inhibition of the proteasome has emerged as an attractive target for anti-cancer therapy. YSYOIA is a novel small molecule compound targeting the proteasome. The compound was found to suppress viability of MCF-7 cells and cause limited cell membrane damage as determined by sulforhodamine $B$ assay (SRB) and CytoTox $96^{\circledR}$ non-radioactive cytotoxicity assay. High-content screening (HCS) further shows that YSY01A treatment induces cell cycle arrest on G2 phase within 24 hrs. Label-free quantitative proteomics (LFQP), which allows extensive comparison of cellular responses following YSY0IA treatment, suggests that various regulatory proteins including cell cycle associated proteins and PI3K/Akt pathway may be affected. Furthermore, YSY01A increases p-CDC-2, p-FOXO3a, p53, p $21^{\text {Cipl }}$ and $p 27^{\text {kipl }}$ but decreases $\mathrm{p}-A k t$, $\mathrm{p}$-ERa as confirmed by Western blotting. Therefore, YSYOIA represents a potential therapeutic for breast cancer MCF-7 by inducing G2 phase arrest via ERa and PI3K/Akt pathways.
\end{abstract}

Key words: YSY01A, PS341, MCF-7, High-content screening, Label-free quantitative proteomics, ERa, PI3K/Akt pathways

\section{Introduction}

The ubiquitin proteasome system (UPS) is crucial for the turnover of proteins by controlling cell cycle, programmed cell death, cell proliferation, survival, adhesion and differentiation ${ }^{1}$. UPS dysregulation is implicated in the underlying molecular pathology of a variety of diseases, including cancer ${ }^{2}$. Ubiquitin and various proteasome have thus become an attractive therapeutic target for treatment of malignancies. In the UPS, several ubiquitin ligases, such as RNF123, have been shown to bind directly to estrogen receptor- $\alpha(E R a)$ and regulate its activity. Besides, a number of studies have suggested that pro- teasome inhibition alters ERa-dependent gene transcription via diverse effects and mechanisms $s^{4,5-8}$. For example, PS341 have been reported that inhibited ERa due to direct transcriptional inhibition and loss of RNA polymerase II recruitment on the ERa gene promote $^{7}$. The ERa, expressed in over two thirds of breast cancers, plays an essential role on tumor growth $^{9-10}$, and blockade of the estrogen action is the mainstay of treatment of ER-positive breast cancer. However, activation of the phosphoinositide 3-kinase (PI3K) pathway occurs frequently in breast cancer. The over-activation of PI3K pathway promotes re- 
sistance to the selective estrogen receptor modulators (SERMs) tamoxifen or aromatase inhibitor letrozole which are the first-line treatment for patients with ERa-positive breast cancer ${ }^{11}$. The molecular mechanism reveals that ERa and the PI3K/Akt pathways form a positive feedback and their cross-talk has also been demonstrated in cancer cells. As the downstream target of PI3K/Akt signaling pathway, the kinase p70S6K can activate ERa by phosphorylation at Ser167 residue. Subsequently, activated ERa acts on PI3K, resulting in a positive feedback loop ${ }^{12,5}$. Inhibition of PI3K pathway and ERa by proteasome inhibitors therefore represents a potentially attractive strategy for the treatment of breast cancer ${ }^{13,14-15}$.

Proteasome inhibitor, as a new anticancer agent, is promising for anticancer therapy that benefit patient with multiple myeloma and non-hodgkin's lymphoma. Bortezomib (also called PS341) have been approved for the treatment of multiple myeloma or relapsed/refractory mantle cell lymphoma ${ }^{16-17}$. These studies also reported that multiple myeloma cell lines that were previously resistant to melphalan, doxorubicin, dexamethasone, or mitoxantrone were sensitized up to 1,000,000-fold by prior exposure to subtoxic concentrations of bortezomib are in clinical development because of their anti-neoplastic and anti-inflammatory 18-19. Bortezomib blocks TNF- $\alpha$ induced NF-kB activation in a dose- and time-dependent manner in multiplemyeloma

cells through degradation of IKBa ${ }^{20}$. Carfilzomib, the second generation proteasome inhibitor, is a more selective proteasome inhibitor with more mild toxicity compared to bortezomib and is also able to overcome resistance to chemotherapeutic agents ${ }^{21}$. Besides, there are some proteasome inhibitors are under investigation, such as Marizomib (NPI-0052), Ixazomib (MLN9708), which are safety and efficacy in some patients from both phase I and II trials ${ }^{22}$. However, their antitumor activity remains unsatisfactory, especially in most solid tumors such as breast cacner ${ }^{23-24}$. Herein, there is considerable interest in seeking a proteasome inhibitor for the treatment of solid tumor malignancies.

Recently, our collaborator, Dr. RT Li, identified a novel proteasome inhibitor, YSY01A, we have demonstrated that YSY01A has less toxicity to livers, kidneys and intestines of nude mice and ICR mice compared with PS341 (data to be published). In this study, we further investigate inhibitory effect of YSY01A on tumor cell viability and find that the compound induces cell cycle arrest in MCF-7 cells. Together, we conclude that YSY01A promises a novel probe for development of proteasome inhibitors and a potential therapeutic for breast cancer treatment.

\section{Materials and methods}

\section{Cell Culture}

Human breast cancer cells MCF-7 were maintained as previously described ${ }^{25}$. For all experiments, cells were cultured in RPMI-1640 medium (Macgene, China) supplemented with $10 \%$ fetal bovine serum, $100 \mathrm{U} / \mathrm{ml}$ penicillin, and $100 \mu \mathrm{g} / \mathrm{ml}$ streptomycin (Macgene, China) in a $37^{\circ} \mathrm{C}$ humidified atmosphere containing $5 \% \mathrm{CO}_{2}$ (Heraeus, Germany).

\section{Cell Viability Assay}

Cell viability and cytotoxicity of YSY01A to MCF-7 cells was determined using sulforhodamine B (SRB) colorimetric assay and CytoTox96 ${ }^{\circledR}$ non-radioactive cytotoxicity assay. Briefly, 4,000 cells/well of MCF-7 cells were plated in a 96-well plate overnight. Cells were then treated with vehicle control (PBS), YSY01A (20, 40, $80 \mathrm{nM})$ or PS341 (40 $\mathrm{nM}$ ) for 12, 24 and 48 hrs (SRB assay) or 24, 48 and 96 hrs (CytoTox96 ${ }^{\circledR}$ assay), respectively. SRB assay was carried out as previously described. ${ }^{25}$ For CytoTox $96^{\circledR}$ assay, cells were firstly lyzed following the manufacturer's instructions for determination of the maximum LDH release. On the other side, released LDH in culture medium of parallel groups was measured after brief spin down and harvest for detection of cell damage following treatments. The percentage of cellular injury was calculated using the formula: \% cytoxicity $=$ experimental LDH released / maximum LDH release $\times 100$ and normalized to vehicle control ${ }^{26}$.

\section{Image Based Quantitative Analysis to Deter- mine Cell Cycle Distribution by HCS}

Click-iT ${ }^{\circledR}$ Alexa Fluor ${ }^{\circledR} 488$ TUNEL imaging assay (Invitrogen, USA) was used to determine the effects of YSY01A on cell cycle. MCF-7 cells were seeded overnight in a 96 well imaging plate followed by treatment with 20, 40 and $80 \mathrm{nM}$ YSY01A for $24 \mathrm{hrs,}$ and then fixed with EdU (5-ethynyl-2'-deoxyuridine) for $1 \mathrm{hr}$ and labeled with Alexa Fluor ${ }^{\circledR} 488$ probe or labeled with antibody against phospho-histone 3 (p-H3). Cells were also co-stained with Hoechst 33342 dye to determine the DNA content based on the nuclear intensity and finally analyzed by high-content screening system equipped with Columbus analysis tool (Cellomics, USA). Cell cycle distribution was defined to $S$ phase by incorporation of the thymidine analog, EdU, and M phase by immunostaining for the mitotic marker, p-H3, while the remaining unlabeled cells were identified to undergo G1 or G2 phases $^{27}$.

\section{D LC-MS/MS Label-free Quantitative Pro- teomic Analysis}

2D LC-MS/MS label-free quantitative proteomic 
analysis was used to investigate the molecular profiles of YSY01A in MCF-7 cells. To prepare cell culture samples, MCF-7 cells were exposed to vehicle control (PBS), YSY01A or PS341 for $24 \mathrm{hrs}$ and lysed in ice-cold lysis buffer $(8 \mathrm{M}$ Urea, $75 \mathrm{mM} \mathrm{NaCl}, 50 \mathrm{mM}$ Tris, protease inhibitor cocktail, $\mathrm{pH}$ 8.2) followed by sonication and high-speed centrifugation at 14,000 rpm for 30 mins. The resulting supernatants were harvested and their protein concentrations were measured using bicinchoninic acid protein assay (Dingguo Changsheng Biotechnology, China). Prior to proteomic analysis, protein samples were reduced by incubation with DTT at a final concentration of $5 \mathrm{mM}$ for 25 mins at $56^{\circ} \mathrm{C}$ and then alkylated by $14 \mathrm{mM}$ iodoacetamide for 30 mins in the dark. Unreacted iodoacetamide was quenched by adding $0.5 \mathrm{M}$ DTT and incubated for 15 mins at room temperature in the dark, and the protein mixture was subsequently diluted 1:5 in $25 \mathrm{mM}$ Tris- $\mathrm{HCl}, \mathrm{pH} \mathrm{8.2,} \mathrm{to} \mathrm{reduce} \mathrm{the}$ concentration of urea to $1.6 \mathrm{M}$. $\mathrm{CaCl}_{2}$ was added to a final concentration of $1 \mathrm{mM}$ with addition of trypsin at $37{ }^{\circ} \mathrm{C}$ overnight. The digestion was stopped by acidification with $0.4 \%$ (vol/vol) trifluoroacetic acid followed by $\mathrm{pH}$ adjustment to 2.0 and centrifugation at $2,500 \mathrm{~g}$ for $10 \mathrm{mins}$ at room temperature. $200 \mu \mathrm{g}$ of peptides from each sample were separated into 15 fractions by strong anion exchange as described previously $^{28}$. For 2D LC-MS/MS analysis, the eluted peptides were concentrated and purified and separated by reverse-phase chromatography (Xbridge ${ }^{\mathrm{TM}}$ C18 3.5um, 4.6x20mm column, and Xbridge ${ }^{\mathrm{TM}}$ BEH130 C18 3.5um,2.1×150mm column, Waters, Ireland). A nanoflow high performance liquid chromatography (HPLC) system (EASY-column,10 cm,ID 75um,3um, Thermo Fisher, USA) equipped was used to analysis, followed by redissolving in formic acid, and loading onto the column (EASY-column, $2 \mathrm{~cm}$, ID 100um, 5um,C18, Thermo Fisher, USA). The 15 high-intensity peaks were used for MS/MS analysis using LTQ Orbitrap velos Pro. Finally, MaxQuant software version 1.4.1.2 was used for data analysis as previously described ${ }^{29-30}$.

\section{Western Blotting}

The expression of proteins of interest was evaluated by Western blotting as we described previous$1 y^{31}$. Whole cell lysates were extracted and separated on SDS-PAGE, followed by immunoblotting against antibodies. Immunoreactive bands were visualized using Bio-Rad system (Bio-Rad, USA) with enhanced chemiluminescence (Bio-Rad).

\section{Statistical Analysis}

Data are presented as the mean $\pm S D$ and analyzed by Student's t-test for comparison of two groups, or by one-way analysis of variance (ANOVA) followed by Tukey's post-hoc test for multiple comparison. Statistical significance was considered at ${ }^{*} p<0.05,{ }^{* *} p<0.01,{ }^{* * *} p<0.001$.

\section{Results}

\section{YSY01A suppresses MCF-7 cells survival}

To determine whether YSY01A suppresses the survival of human breast cancer cells MCF-7, the cell viability was assessed by SRB assay following treatment with 20, 40 and $80 \mathrm{nM}$ YSY01A for 12, 24 and 48 hrs (Fig. 1A). The compound inhibited survival of MCF-7 cells in concentration- and time- dependent manners with significant difference observed at 24 and 48 hrs. Compared to vehicle control (PBS), 20, 40 and $80 \mathrm{nM}$ YSY01A were found to reduce cell survival by $11.6 \%, 20.8 \%$ and $32.0 \%$ at 24 hrs as well as $26.8 \%$, $44.2 \%$ and $66.4 \%$ at $48 \mathrm{hrs}$, respectively ( $\mathrm{p}<0.001 \mathrm{ex}-$ cept for $11.6 \%$ ). To further detect the cytotoxicity of YSY01A, LDH (lactate dehydrogenase) release, indicative of rupture of cell membranes following treatment, was measured in MCF-7 cells exposed to 20, 40 and $80 \mathrm{nM}$ YSY01A for 24, 48 and 96 hrs. The results revealed that treatment with $80 \mathrm{nM}$ YSY01A for $96 \mathrm{hrs}$ raised LDH release by $69.7 \%$ and became 2.31 fold with statistical significance as compared with vehicle control ( $p<0.01)$. At 24 and 48 hrs, although the LDH release increased in a concentration- and time-dependent manner, significant difference was not observed between YSY01A and vehicle control (Fig. 1B), suggesting that YSY01A inhibited the survival of MCF-7 cells with limited cell damage, so we speculate that cell cycle progression may be susceptible for YSY01A treatment.

\section{YSY01A induces cell cycle arrest on G2 phase in MCF-7 cells}

To investigate whether cell cycle arrest may contribute to loss of cell viability after YSY01A treatment, we further performed a cell cycle analysis using HCS. As shown in Fig. 2, increasing concentrations of YSY01A resulted in a dose-dependent rise in the relative NINI (normalized integrated nuclear intensity) value that corresponds to $\mathrm{G} 2$ phase. Exposure to YSY01A for $24 \mathrm{hrs}$ caused an accumulation of cells on G2 phase from $28.6 \%$ (PBS) to $36.0 \%$ (40 nM) and $41.8 \%$ (80 nM) (Table 1). Taxol (Tax), which stabilizes microtubules by reducing their dynamicity and promotes mitotic arrest, was used as a positive control. Exposure to $100 \mathrm{nM}$ Taxol caused M phase arrest in MCF-7 cells as determined by an increase in the NINI values. Thus, cell cycle arrest on G2 phase probably contributes to suppression of cancer cell survival in MCF-7 cells. 
A

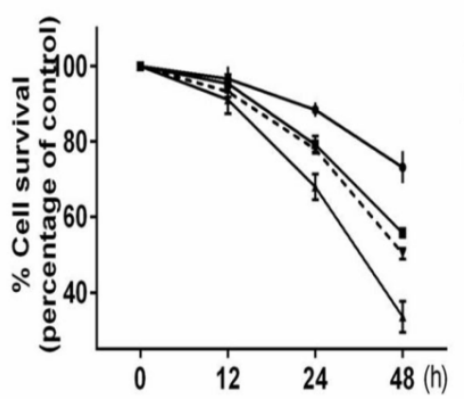

B

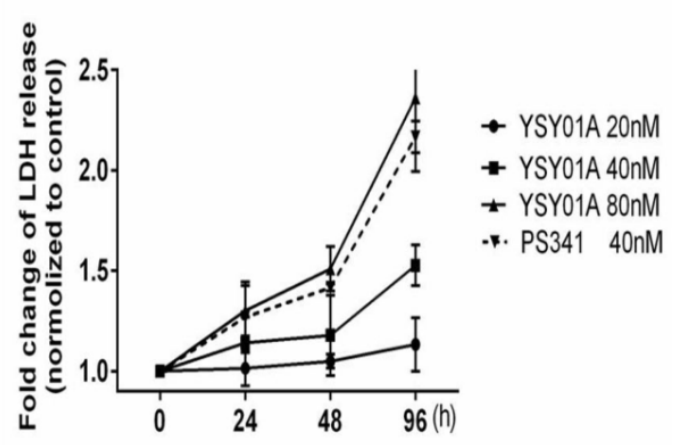

Figure 1. YSY01A inhibits survival of MCF-7 cells. MCF-7 cells were treated with either the vehicle (PBS), YSY01A 20, 40, $80 \mathrm{nM}$ or PS341 $40 \mathrm{nM}$ for 12,24 and 48 hrs (A) or 24,48 and 96 hrs (B) followed by sulforhodamine B (SRB) assay (A) or CytoTox $96 \AA$ assay (B). Data are represented as the mean of three independent experiments performed in triplicate. $* * * p<0.001$

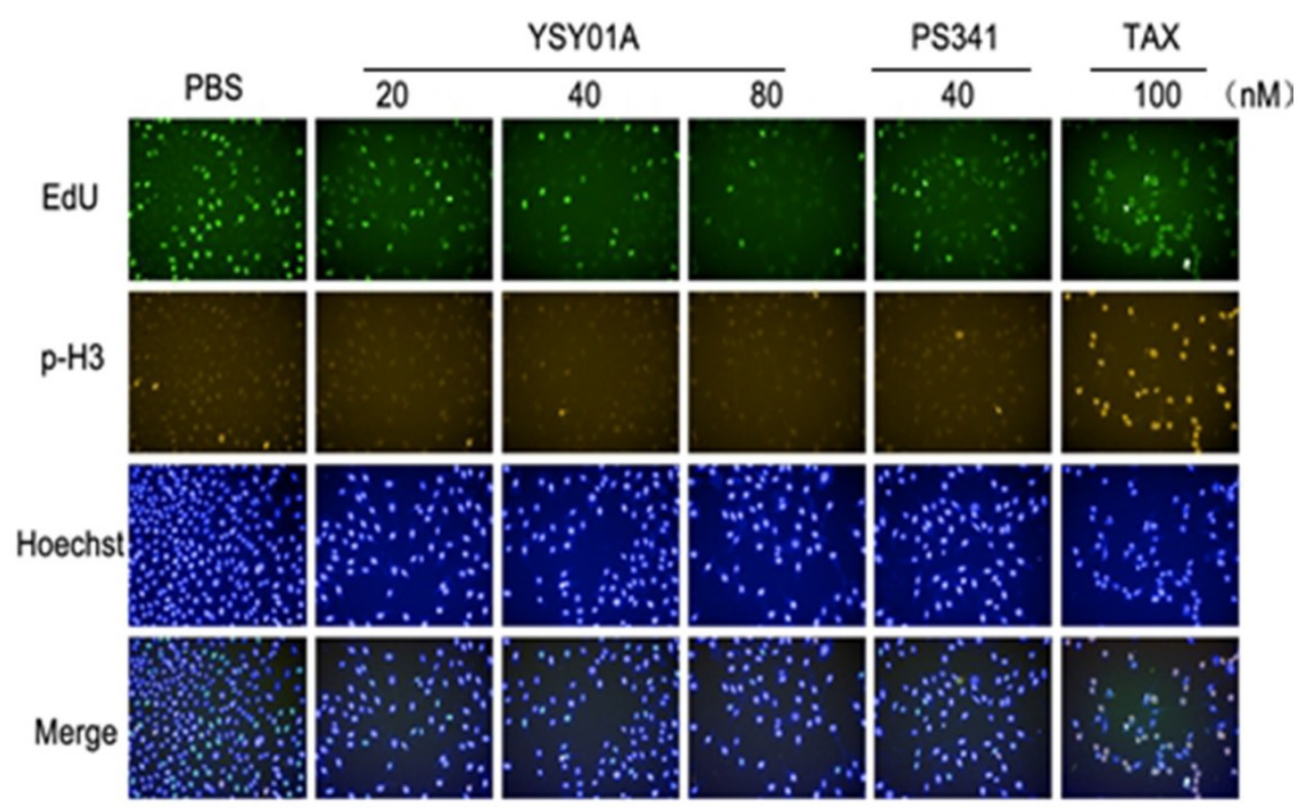

Figure 2. YSY01A induces cell cycle arrest in MCF-7 cells as determined by high-content imaging. Representative images of MCF-7 cells stained with EdU (5-ethynyl-2'-deoxyuridine)(a marker of S phase), p-H3 (a marker of M phase) and Hoechst 33342 (a marker of DNA), and the merge image also were shown. TAX represented the microtubules stabilizer taxol.

Table 1. Percentage induction cell cycle arrest in MCF-7

\begin{tabular}{llrrrr}
\hline Group & $\begin{array}{c}\text { Concentration } \\
(\mathrm{nM})\end{array}$ & \multicolumn{4}{c}{$\begin{array}{c}\text { \% Percentage of cell cycle distribution } \\
(\text { mean } \pm \text { SD) }\end{array}$} \\
\cline { 3 - 6 } & & $\mathrm{G} 1$ & $\mathrm{~S}$ & $\mathrm{G} 2$ & $\mathrm{M}$ \\
\hline PBS & 0 & $26.8 \pm 4.7$ & $43.3 \pm 3.8$ & $28.6 \pm 1.9$ & $1.5 \pm 0.6$ \\
YSY01A & 20 & $17.2 \pm 1.3$ & $53.9 \pm 1.8$ & $28.3 \pm 0.8$ & $0.5 \pm 0.2$ \\
& 40 & $34.1 \pm 1.6$ & $28.1 \pm 3.9$ & $36.0 \pm 4.5$ & $1.7 \pm 0.8$ \\
& 80 & $18.6 \pm 0.2$ & $38.8 \pm 1.2$ & $41.8 \pm 1.4^{* *}$ & $0.8 \pm 0.0$ \\
PS341 & 40 & $21.9 \pm 5.5$ & $46.9 \pm 0.5$ & $30.9 \pm 4.8$ & $0.3 \pm 0.2$ \\
TAX & 100 & $22.9 \pm 3.9$ & $32.4 \pm 4.3$ & $28.9 \pm 5.1$ & $15.6 \pm 6.2^{*}$ \\
\hline
\end{tabular}

YSY01A induces cell cycle arrest in MCF-7 cells as determined by high-content imaging. Cells were treated in triplicate at noted concentrations of YSY01A for $24 \mathrm{~h}$. Distribution percentage of cell cycle phases based on NINI (normalized integrated nuclear intensity values) derived from the DNA binding dye
(Hoechst 33342). NINI value is calculated by measuring the integrated nuclear intensity divided by the number of nuclei within each well. The NINI derived from mitotic nuclei ( $\mathrm{p}-\mathrm{H} 3$ labeled) were indicative of $4 \mathrm{~N}$ DNA content, while NINI values for S phase (EdU labeled nuclei) were ranged between 0.5 and $1 X$ NINI values of $\mathrm{p}-\mathrm{H} 3$ ( $2 \mathrm{~N}$ to $4 \mathrm{~N}$ DNA content), and images were analyzed using Columbus software. ${ }^{*} p<0.05$, ${ }^{* *} p<0.01$.

\section{YSY01A affects PI3K pathway and cell cycle associated proteins}

$\mathrm{PI} 3 \mathrm{~K}$ and cell cycle associated proteins were changed significantly by YSYO1A in LFQP assay

To extensively explore molecular mechanisms of YSY01A, LFQP offers a robust means for obtaining proteome profiles of YSY01A (Fig. 3A). The results 
showed that YSY01A was involved in regulation of apoptosis pathway, p53 associated pathway, mitochondrial dysfunction, endoplasmic reticulum stress, ubiquitin pathway, etc. PI3K pathway and cell cycle associated proteins were two of the most valuable and dominant fraction affected. The information of all proteins in the presence of $40 \mathrm{nM}$ dose of YSY01A and PS341 were identified (Additional file 2: Supplemental data), and 395 peptides of 7560 whose abundances changed by more than two folds were identified (Fig. 3A\&B). As shown in Fig. 3C, CDKN2A (p16), CDKN1A (p21 ${ }^{\text {Cip1) }}$ ) became 6.6 and 6.2 fold compared to vehicle control following exposure to YSY01A, while G2/M phase markers, CCNB1 (Cyclin B1) and CCNB2 (Cyclin B2), showed a 2.7 and 2.3 fold increase. Kinases in G2/M phase including CDC-6, CDC-20 and BUB1 exhibited a rise of 4.1, 2.6 and 2.3 fold, respectively, and YSY01A also increased levels of Wee1 to 2.2 fold. Sestrin 2 (SESN2), provides an important link between genotoxic stress, p53 and the mTOR signaling pathway, showed a 3.6 fold increase. And the profiles of PS341 were roughly coincident with YSY01A, apart from more intense enhancement in p16, p21 Cip1 (6.9 and 8.8 fold change) and more mild increase in Wee1 (1.8 fold) compared with YSY01A. Moreover, quantitative analysis indicated that YSY01A may regulate PI3K pathway. For example, PIK3C3, Akt1 and PDK2 declined by $25.6 \%, 14.8 \%$, $38.7 \%$ and p70S6K (RPS6KB1) decreased by $29.2 \%$, whereas Deptor, an upstream inhibitor of mTORC1, was increased by 3.9 fold. The profiles of PS341 were also consistent with YSY01A except a slight increase in Deptor by 1.20 fold. These results were indicative of the potential involvement of YSY01A in regulation of cell cycle progression and PI3K pathway.

\section{YSY01A regulates expression of cell cycle, PI3K/Akt associated proteins and ERa}

To validate molecular profiles of YSY01A identified by LFPQ, the G2/M phase marker, Cyclin B, CDC-2 and p-CDC-2 were firstly assessed by Western blotting. As shown in Fig. 4A, Cyclin B, CDC-2 and p-CDC-2 were all increased in MCF-7 cells treated with YSY01A, PS341 showed a similar trend as YSY01A with a slight increase in Cyclin B, and the similar results were obtained following YSY01A treatment at different time (Additional file 1: Fig. S1A). For the pivotal proteins involved in cell cycle transition, p53 were increased following YSY01A treatment, and the expression of p21 ${ }^{\mathrm{Cip} 1}$ and p27Kip1 increased in the presence of $40 \mathrm{nM}$ and $80 \mathrm{nM}$ YSY01A (Fig. 4B). Moreover, it was not difficult to find that the bands began increasing at $3 \mathrm{hrs}$ and reached a peak at 24 hrs (Additional file 1: Fig. S1B). However, the level of FoxO3a was decreased while its phosphorylation level was increased significantly. There was no obvious difference between PS341 and YSY01A, except PS341 treatment showed a more dramatic alternation in p53 and p27Kip1 than YSY01A. The increase of p21 ${ }^{\text {Cip1 }}$, p27Kip1 and p53 expression and activity caused by YSY01A suggested that the molecular change was significant for cell cycle arrest following YSY01A treatment.
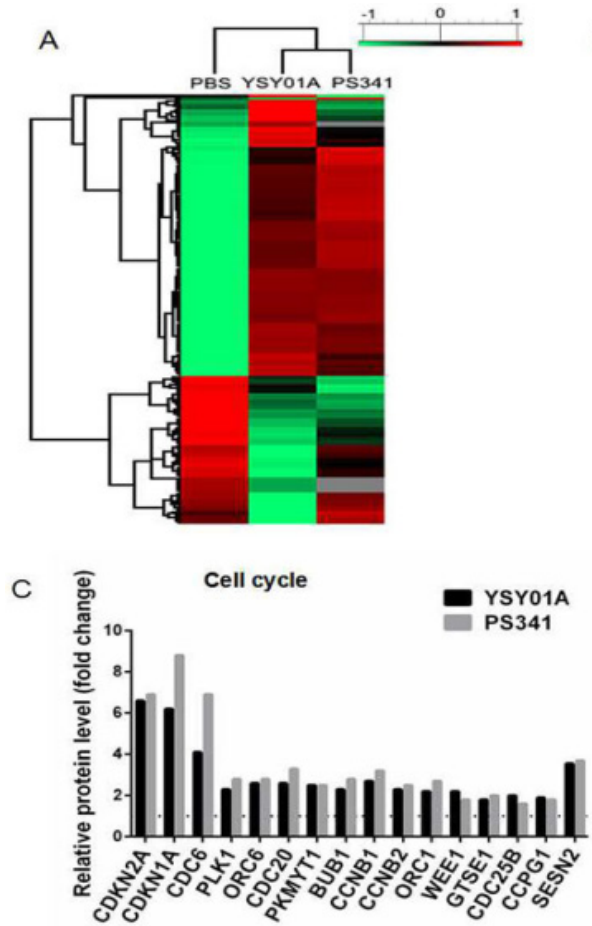
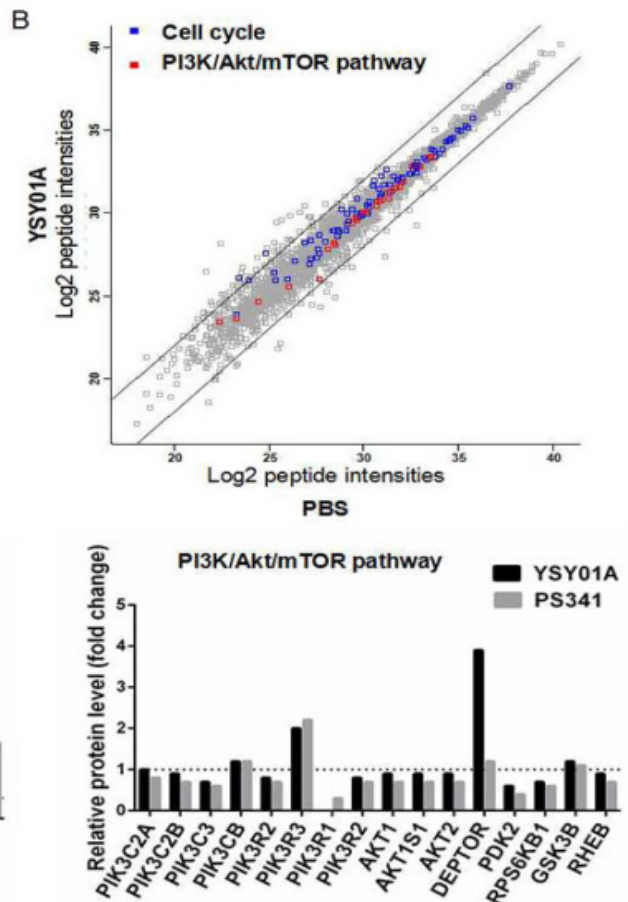

Figure 3. YSYOIA changes PI3K and cell cycle associated proteins in MCF-7 cells revealed by LFQP (label-free quantitative proteomics). (A) Symmetrical matrix/non-clustering heatmap of Pearson correlation coefficients values from the significantly changed peptide intensity. Of 7560 peptides (image not shown in A), 395 showed abundance change of more than two folds. Normalized protein abundance values (Z-scores) were indicated colorimetrically for each protein in each sample ranging from green (low correlation, minimum, -1), to black(median, 0), and red (high correlation, maximum 1) (B) Scatter plot of all the log2 peptide intensities plotted against PBS group. The two oblique line showed $Y=X+2$ and $\mathrm{Y}=\mathrm{X}-2$, separating proteins at a ratio of 2 or 0.5 compared to PBS. The blue spots and red spots represented peptides of cell cycle proteins and PI3K/Akt/mTOR pathway. (C) Histograms of relative peptides quantitative levels of cell cycle and PI3K/Akt/mTOR pathway. The value of PBS was defined as 1 and indicated by a dash line. Sets of LC-MS/MS data were collected in triplicate following YSY01A treatment of $24 \mathrm{hrs}$. 


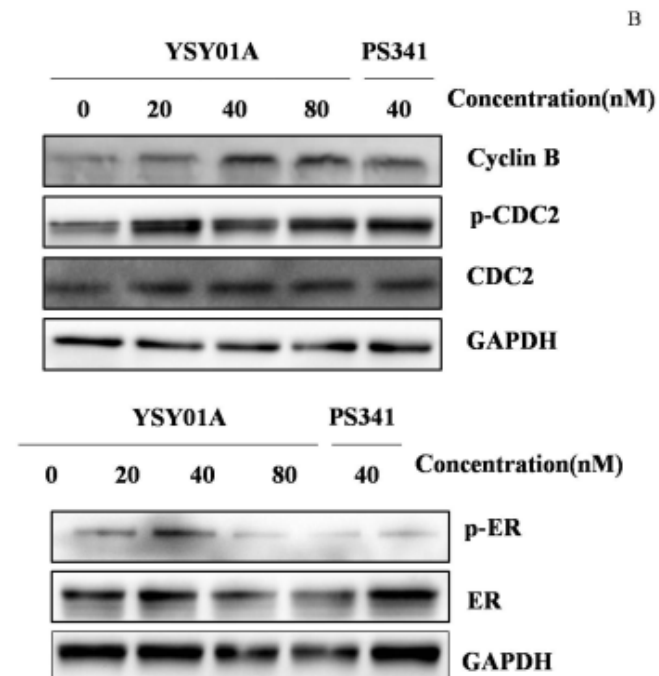

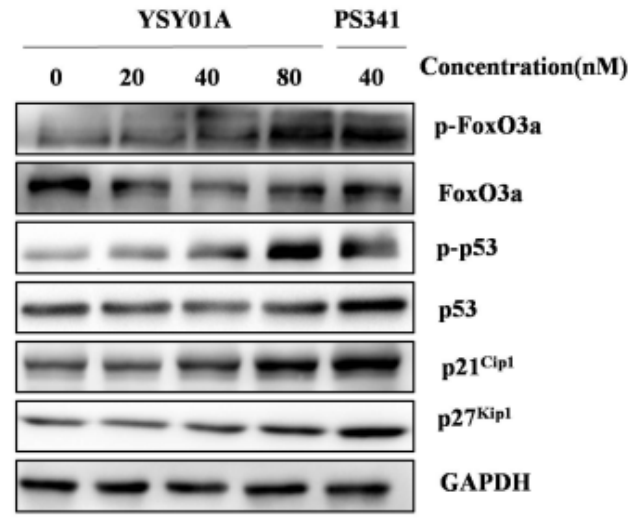

Figure 4. YSYO1A induces pivotal proteins involved in cell cycle transition and ERa in MCF-7 cells. After treatment of 20, 40, $80 \mathrm{nM}$ YSY01A for $24 \mathrm{hrs}$ or $40 \mathrm{nM}$ PS341 for 24 hrs, expression of the cell cycle markers (A), cell cycle proteins (B) and ERa (C) were evaluated. Cell lysates were fractionated on SDS-polyacrylamide gels and analyzed by Western blotting with antibodies against cell cycle associated proteins. GAPDH was used as a loading control.

A

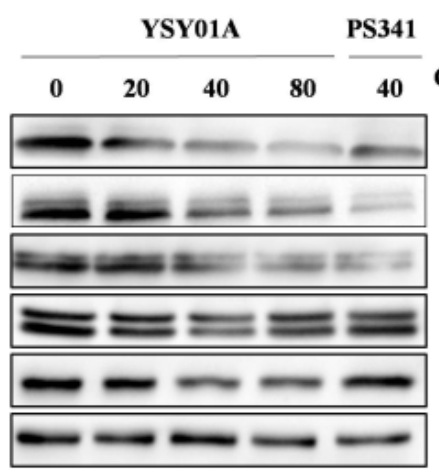

B

YSY01A PS341 L

\begin{tabular}{|c|c|c|c|c|c|c|}
\hline $\mathbf{0}$ & 20 & 40 & 80 & 40 & 100 & Concentration(nM) \\
\hline- & $=$ & - & - & $=$ & $=$ & p-mTOR \\
\hline- & $=$ & 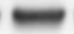 & $=$ & $=$ & 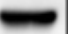 & mTOR \\
\hline $\mathbf{9}$ & $m$ & $=$ & $m$ & $=$ & $=0$ & p-p70S6K \\
\hline- & $\longrightarrow$ & - & $=$ & - & $=$ & p70S6K \\
\hline- & $=$ & $=$ & $=$ & $x=$ & - & Deptor \\
\hline- & - & - & - & $=$ & - & GAPDH \\
\hline
\end{tabular}

Figure 5. YSY01A modulates expression of PI3K pathway-related proteins. MCF-7 cells were treated with YSY0IA at 20, $40,80 \mathrm{nM}$ for $24 \mathrm{hrs}$ as directed and then subject for Western blotting analysis. $L$ represented the positive control medicine rapamycin. GAPDH was used as a loading control.

Considering the potential role of YSY01A in regulation of PI3K pathway, the phosphorylation of Akt, an activator of mTOR, and its downstream effector p70S6K were also evaluated by Western blotting in MCF-7 cells following YSY01A treatment. As shown in Fig. 5A, 40 and 80 nM YSY01A significantly inhibited the phosphorylation of Akt (Ser473 and Thr308), and the inhibition appeared starting a treatment of 3 hrs (Additional file 1: Fig. S2A). Furthermore, Deptor, a depressor of mTOR, was definitely raised after exposure to 40 and $80 \mathrm{nM}$ YSY01A for 24 hrs, and $40 \mathrm{nM}$ PS341 induced the same outcome as well (Fig. 5B \& S2C). Since Akt promotes cell cycle progression, we speculate that the decreased phosphorylation level of Akt may result in cell cycle arrest in MCF-7 cells treated withYSY01A or PS341.

Additionally, Western blotting revealed that ERa and p-ERa was down-regulated by YSY01A and PS341 (Fig. 4C \& S1C). ER $\alpha$ is frequently overexpressed in the early stage of breast cancer and com- mitted to modulate cell cycle progression, suggesting the response of ER-positive cells to YSY01A.

\section{Discussion}

The UPS plays a vital role in many cellular functions by selectively degrading key regulatory proteins. By blocking the action of proteasomes, proteasome inhibitors modulates cellular complexes that break down proteins and have been studies in anti-tumor treatment. Pre-clinical studies have revealed that bortezomib can sensitize the responses of cancer cells to chemotherapy agents and/or radiation therapy, for example 5-fluorouracil, cisplatin ${ }^{32}$, however, its toxicity prevents the achievement of higher drug concentrations and thus limits the efficacy of bortezomib monotherapy in certain cancers. Carfilzomib is a more selective proteasome inhibitor that is structurally distinct from bortezomib with reduced peripheral neuropathy. United States Food and Drug Administration granted authorization to carfilzomib in 2012 
for treatment of multiple myeloma ${ }^{33}$. Pre-clinical data also support future clinical evaluation of carfilzomib in B-cell lymphoma ${ }^{34}$. However, the present proteasome inhibitors are usually ineffective to most solid tumors, including breast cancer. The new proteasome inhibitor, YSY01A, represents our attempt to develop more potent and less toxic proteasome inhibitors, and available for more wide variety of tumors including solid tumor. Previous studies have demonstrated little toxicity of YSY01A in the tumor-bearing nude mice (data to be published). In this study, we found that YSY01A inhibits cell vitality and induces cell cycle arrest in human breast cancer cell line MCF-7, suggesting its potential use in antitumor therapy.

With the aid of LFQP, the molecular profiles of YSY01A was revealed as well. LFQP assay allows analysis of abundant data about the changes of proteins levels following YSY01A treatment via a robust and easy-to-implement label-free relative quantification approach. This technique also obviate the requirements of protein staining or peptide labeling ${ }^{35}$. We identified a total of 7,561 non-redundant proteins from the 15 highest peaks in MS/MS, indicative of 52 categories changed, including the ubiquitination process, protein degradation (proteasome), mTOR, ErbB, cell cycle, and apoptosis. However, this assay could cause an omission of low-abundance proteins that may play an important role in cells' life. In relative to the overall blueprint of intracellular proteins, Western blotting was used to validate the results of LFQP and make an excellent complement of the alteration of some low-level proteins.

Additionally, the combination therapy involving cyclophosphamide, doxorubicin, and 5-fluorouracil (5-FU) (CDF) is the most common chemotherapeutic regimen of breast cancer ${ }^{36}$. Nevertheless, the clinical usefulness of CDF is limited by its remarkably low therapeutic window and frequent eruption of drug resistance. Therefore, development of chemopreventive and therapeutic agents is needed to provide more therapeutic options, especially specific to drug resistance caused by PI3K/Akt over-expression. It has been demonstrated that blockade of the PI3K/Akt pathway can produce profound alternations in downstream gene expression ${ }^{37}$, including p53, FoxO, p21, p27. It is worth to note that some reports are demonstrated that PS341 decreases drug resistance via altering DNA repair pathways ${ }^{38}$ and histone deacetylase ${ }^{39-40}$ in relapsed/refractory multiple myeloma. Moreover, a phase II study of the combination of endocrine treatment and bortezomib in patients revealed that resistance to anti-hormonal therapies may be the result of an activated NF- $\mathrm{KB}$ signalling pathway in breast cancer ${ }^{41}$. NF- $\mathrm{kB}$ signalling pathway was not significantly altered after YSY01A and bortezomib treatment in our LFQP assay and the previous study of our group[data to be published]. The main reason may be associated with the status of $\mathrm{NF}-\mathrm{kB}$, as NF- $\mathrm{kB}$ was enhanced when the cancer cells obtain the drug resistance ${ }^{42-43}$, and the next plan in our group will focus on the effect of YSY01A on drug resistance when combined with other drugs.

$E R a$ is another important therapeutic target in ERa-positive cancer cells, and a number of studies have reported the ERa alteration with bortezomib treatment. In addition to the previously mentioned studies, ${ }^{3-8}$ bortezomib has been also found to cause a strong increase in p21 ${ }^{\text {Cip } 1}$ mRNA level because of inhibit the ERa expression in ERa-positive cells ${ }^{44}$. In our study, YSY01A significantly enhanced the expression of p21 $^{\text {Cip } 1}$ and relatively slightly altered the status of ERa expression, although the activity of ERa (p-ERa) was significantly inhibited by YSY01A and PS341. For the reason, the positive feedback between ERa and p70S6K point to the potential cause, indeed, we observed that ERa and p70S6K activity were declined in MCF-7 cells following YSY01A and PS341 treatment.

In conclusion, our study reinforces the potential benefit of YSY01A in cancer treatment, and provides extensive description of the mechanism of YSY01A on cell cycle arrest. We suggest that YSY01A may inhibit cell cycle progression and up-regulate the tumor suppressor genes $\mathrm{p} 53$, $\mathrm{p} 27^{\mathrm{kip} 1}$ and $\mathrm{p} 21^{\mathrm{Cip} 1}$ by inhibition of PI3K/Akt pathway and ERa activity in MCF-7 cells. Further studies directed at this strategy will provide more insights into the approach to interrupt proteasome for potential antineoplastic therapy.

\section{Supplementary Material}

Additional File 1:

Figures S1-S2.

http://www.jcancer.org/v06p0319s1.pdf

Additional File 2:

Supplemental data.

http://www.jcancer.org/v06p0319s2.xlsx

\section{Acknowledgments}

Funding for this project was provided by the Ministry of National Science and Technology National Major Scientific and Technological Special Project for "Significant New Drugs Development" Program (No. 2009 ZX0930010) and the State Key Program of National Natural Science Foundation of China (No. 81172915).

\section{Competing Interests}

The authors have declared that no competing interest exists. 


\section{References}

1. D'Arcy P, Linder S. Molecular pathways: translational potential of deubiquitinases as drug targets. Clin Cancer Res 2014; 20(15):3908-14.

2. Liu YB, Gao X, Deeb D, et al. Ubiquitin-proteasomal degradation of antiapoptotic survivin facilitates induction of apoptosis in prostate cancercells by pristimerin. Int J Oncol 2014; 45(4):1735-41.

3. Johnsen SA, Gungor C, Prenzel T, et al. Regulation of estrogen-dependent transcription by the LIM cofactors CLIM and RLIM in breast cancer. Cancer Res 2009; 69(1):128-136.

4. Fan M, Nakshatri H, Nephew KP. Inhibiting proteasomal proteolysis sustains estrogen receptor-alpha activation. Mol Endocrinol 2004; 18(11):2603-15.

5. Lonard DM, Nawaz Z, Smith CL, O'Malley BW. The $26 \mathrm{~S}$ proteasome is required for estrogen receptor-alpha and coactivator turnover and for efficient estrogen receptor-alpha transactivation. Mol Cell 2000; 5:939-48.

6. Reid G, Hubner MR, Metivier R, et al. Cyclic, proteasome-mediated turnover of unliganded and liganded ERa lpha on responsive promoters is an integral feature of estrogen signaling. Mol Cell 2003; 11(3):695-707.

7. Powers GL, Ellison-Zelski SJ, Casa AJ, et al. Proteasome inhibition represses ERa gene expression in ER+ cells- a new link between proteasome activity and estrogen signaling in breast cancer. Oncogene 2010; 29(10): 1509-1518.

8. Zhang H, Sun L, Liang J, et al. The catalytic subunit of the proteasome is engaged in the entire process of estrogen receptor-regulated transcription. EMBO J 2006; 25:4223-33.

9. Deroo BJ, Korach KS. Estrogen receptors and human disease. J Clin Invest 2006; 116(3):561-70.

10. Ali S, Coombes RC. Endocrine-responsive breast cancer and strategies for combating resistance. Nat Rev Cancer 2002; 2(2):101-12.

11. Josh Lauring, Ben Ho Park, Antonio C. Wolff. The Phosphoinositide-3-Kinase-Akt-mTOR Pathway as a Therapeutic Target in Breast Cancer. J Natl Compr Canc Netw 2013; 11(6):670-678.

12. Sanchez CG, Ma CX, Crowder RJ, et al.. Preclinical modeling of combined phosphatidylinositol-3-kinase inhibition with endocrine therapy for estrogen receptor-positive breast cancer. Breast Cancer Res 2011; 13(2):R21.

13. Mahadevan D., Chiorean E.G., Harris WB., et al. Phase I pharmacokinetic and pharmacodynamic study of the pan-PI3K/mTORC vascular targeted pro-drug SF1126 in patients with advanced solid tumours and B-cell malignancies. Eur. J. Cancer 2012; 48(18):3319-3327.

14. Liu CY, Shiau CW, Kuo HY, et al. Cancerous inhibitor of protein phosphatase 2A determines bortezomibinduced apoptosis in leukemia cells. Haematol 2013; 98(5):729-738.

15. Chen KF, Yeh PY, Yeh KH, et al. Down-regulation of phospho-Akt is a major molecular determinant of bortezomib-induced apoptosis in hepatocellular carcinoma cells. Cancer Res 2008; 68(16):6698-6707.

16. Kane RC, Dagher R, Farrell A, et al. Bortezomib for the treatment of mantle cell lymphoma. Clin Cancer Res 2007; 13(18 Pt 1): 5291-5294.

17. Morra E. Carfilzomib: a new opportunity for WM patients.Blood. Blood 2014; 124(4):468-9.

18. Mohty M, Brissot E, Savani BN, Gaugler B. Effects of bortezomib on the immune system: a focus on immune regulation. Biol Blood Marrow Transplant 2013; 19(10):1416-20.

19. Hideshima T, Richardson P, Chauhan D, et al. The proteasome inhibitor PS-341 inhibits growth, induces apoptosis, and overcomes drug resistance in human multiple myeloma cells. Cancer Res 2001; 61(7):3071-3076.

20. Julian Adams. THE PROTEASOME: A SUITABLE ANTINEOPLASTIC TARGET. Nat Rev Cancer 2004; 4(5):349-60.

21. Nooka AK. Management of Hematologic Adverse Events in Patients With Relapsed and/or Refractory Multiple Myeloma Treated With Single-Agent Carfilzomib. Oncology (Williston Park) 2013; 1:27.

22. Sandra E. Kurtin, Elizabeth Bilotti. Novel Agents for the Treatment of Multiple Myeloma: Proteasome Inhibitors and Immunomodulatory Agents. J Adv Pract 2013; 4(5):307-321.
23. Engel $\mathrm{RH}$, Brown JA, Von Roenn JH, et al. A phase II study of single agent bortezomib in patients with metastatic breast cancer: a single institution experience. Cancer Invest 2007; 25(8):733-737.

24. Dees EC, Orlowski RZ. Targeting the ubiquitin-proteasome pathway in breast cancer therapy. Future Oncol 2006; 2(1):121-135.

25. Xu-Hua Wang, Bo Xu, Jing-Tao Liu, Jing-Rong Cui. Effect of $\beta$-escin sodium on endothelial cells proliferation, migration and apoptosis. Vascular Pharmacology 2008; 49:158-165.

26. Ma W, Chen M, Kaushal S, et al. PLGA nanoparticle-mediated delivery of tumor antigenic peptides elicits effective immune responses. International Journal of Nanomedicine 2012; 7:1475-1487.

27. Kota KP1, Benko JG, Mudhasani R, et al. High Content Image Based Analysis Identifies Cell Cycle Inhibitors as Regulators of Ebola Virus Infection. Viruses 2012; 4(10):1865-1877.

28. Tarawneh R, Lee JM, Ladenson JH, et al. CSF VILIP-1 predicts rates of cognitive decline in early Alzheimer disease. Neurology 2012; 78(10):709-719.

29. Zimmer JS, Monroe ME, Qian WJ, Smith RD. Advances in proteomics data analysis and display using an accurate mass and time tag approach. Mass Spectrom Reviews 2006; 25(3):450-486

30. Yan Fu, Li-Yun Xiu, Wei Ji, et al. AMT: A Statistical Algorithm for Fast Detection of Protein Modifications From LC-MS/MS Data. Technological Innovation and Resources 2011.

31. Hou L, Xu B, Guo W, et al. Pseudolaric acid B inhibits inducible cyclooxygenase-2 expression via downregulation of the NF-kB pathway in HT-29 cells. J Cancer Res Clin Oncol 2012.

32. Boccadoro, M., Morgan, G. Cavenagh, J. Preclinical evaluation of the proteasome inhibitor bortezomib in cancer therapy. Cancer Cell International 2005; 5(1):18.

33. Andreu-Vieyra C, Berenson JR. Carfilzomib in multiple myeloma. Expert Opin Biol Ther 2014; 29:1-15.

34. Gu JJ, Hernandez-Ilizaliturri FJ, Kaufman GP, et al. The novel proteasome inhibitor carfilzomib induces cell cycle arrest, apoptosis and potentiates the anti-tumour activity of chemotherapy in rituximab-resistant lymphoma. British Journal of Haematology 2013; 162(5):657-669.

35. Elliott MH, Smith DS, Parker CE, Borchers C. Current trends in quantitative proteomics. J Mass Spectrom 2009; 44(12): 1637-1660.

36. Ibrahim AS, Sobh MA, Eid HM, et al. Gingerol-derivatives: emerging new therapy against human drug-resistant MCF-7. Tumour Biology 2014; 4(2):39-47.

37. Engelman JA1, Luo J, Cantley LC. The evolution of phosphatidylinositol 3-kinases as regulators of growth and metabolism. Nature Reviews Genetics 2006; 7(8):606-19.

38. Claire Gourzones-Dmitriev, alboukadel Kassambara, Surinder Sahota, et al. DNA repair pathways in human multiple myeloma. Cell cycle 2013; 12(17):2760-2773.

39. Roman Hajek, David Siegel, Robert Z. Orlowski, et al. The role of histone deacetylase inhibitors in patients with relapsed/refractory multiple myeloma. Leukemia \& Lymphoma 2014; 55(1):11-18.

40. Iida S, Ri M. Determinants of sensitivity to proteasome inhibitors and strategies to overcome acquired resistance tobortezomib in multiple myeloma. Rinsho Ketsueki 2014 Mar; 55(3):304-10.

41. Trinh XB, Sas L, Van Laere SJ, et al. A phase II study of the combination of endocrine treatment and bortezomib in patients with endocrine-resistant metastatic breast cancer. Oncology Reports 2012; 27(3):657-63.

42. Erstad DJ, Cusack JC Jr. Targeting the NF-kB pathway in cancer therapy. Surg Oncol Clin N Am 2013; 22(4):705-46.

43. Li X, Abdel-Mageed AB, Mondal D, Kandil E. The nuclear factor kappa-B signaling pathway as a therapeutic target against thyroid cancers. Thyroid 2013; 23(2):209-18

44. Maynadier M, Shi J, Vaillant $\mathrm{O}$, et al. Roles of estrogen receptor and p21(Waf1) in bortezomib-induced growth inhibition in human breast cancer cells. Molecular Mol Cancer Res 2012; 10(11):1473-81. 